\title{
A reviving templating method for multiple nanostructures
}

\author{
Hui-Hui Li and Shu-Hong Yu*
}

Binary materials, comprised by two dissimilar components in one matrix, already exist in nature from the subatomic to the universal scale with different kinds of forms (e.g., nuclei and electrons, matter and antimatter) [1]. With the progress of nanotechnology, binary materials assembled in nanoscale become an important principle to pursuit innovative functions [2]. Especially when the binary sub-components are in the interplay distance, intimate coupling between these components becomes dominant and endows the macroscopic materials with unique physical and/or chemical phenomena with superior functionalities that cannot be achieved by either of the individual sub-components. Therefore, many strategies, including 'bottom-up' wet-chemicals and 'top-down' physical-approaches, have been intensively explored to synthesize different binary materials [3-7]. However, the resultant binary materials are usually facing limited option of compositions and morphologies, as well as poor controlling of distributions and distances. Therefore, exploiting an effective solution to address these issues and realize the practical implementation is a significant request.

Recently, Lei and colleagues at the Ilmenau University of Technology in Germany reported a concept of binary nanostructuring to tackle this long-standing challenge [8]. They ingeniously employed a unique binary-pore anodized aluminium oxide (AAO) template to successfully realize diverse binary materials on large scale. The most important key of their technique is using a selective etching process to generate the binary-pore template, which possesses not only two sets of pores (e.g., square-shaped and round-shaped pores) but also two barriers located on the opposite side of the template (Fig. 1a). These features enable the researchers to modulate each set of pore size and shape in a high degree of freedom, as well as to deposit different materials in each set of pores separately (Fig. 1b and e).

From the in-situ scanning electron microscope (SEM) investigation and electric field simulation on the as-prepared template, the authors concluded that a combination of electric-field assisted dissolution and plastic oxide flow gives rise to the growth of the unique binary-pore structures (Fig. 1c). Based on this purposed mechanism, they successfully developed ternary- and quadruple-pore templates with the same selective etching process. More interestingly, the morphology of the new etched pores, like C-pores in the ternary-pore template, can be adjusted not only by the selective etching time but also by the size difference of A-pore and B-pore. Therefore, with the size difference of A-pore and B-pore decreasing, the shape of C-pores can gradually change from oval to round (Fig. 1d). Considering that their binary-pore templates are also realizable in a wide range of interpore distance from $142 \pm 13$ to 573 $\pm 27 \mathrm{~nm}$, the capability of the binary-pore template is far more beyond than those of the state-of-art hard and soft templates [9].

Combining with well-established approaches, like electrochemical deposition and atomic layer deposition, zeroand one-dimensional binary materials with different shapes, sizes, and compositions have also been synthesized (Fig. 1e). The resultant binary materials are highly dependent on the features of binary-pore template, and countless binary materials are expected using suitable fabrication strategies. To show the potential of the binary nanostructuring concept in puzzling model systems of significant current interests, the authors demonstrated their potential applications in wireless photosynthesis cells, plasmonic de-

Division of Nanomaterials \& Chemistry, Hefei National Laboratory for Physical Sciences at the Microscale, Department of Chemistry, University of Science and Technology of China, Hefei 230026, China

*Email: shyu@ustc.edu.cn 
$\mathbf{a}$

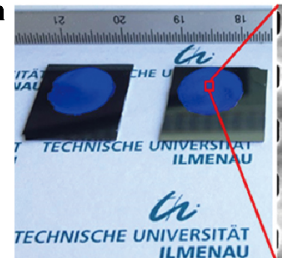

ILMENAU

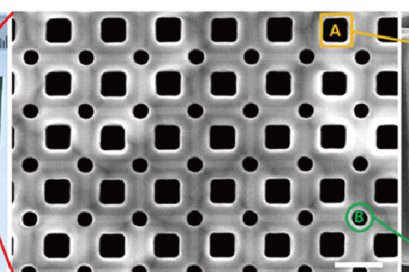

$\mathbf{C}$
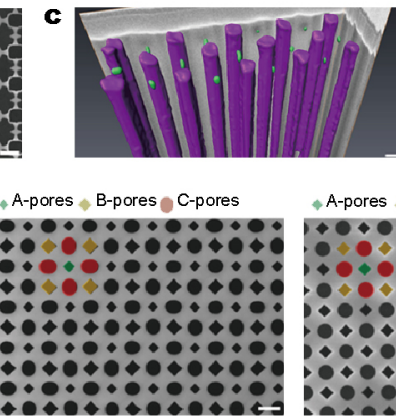

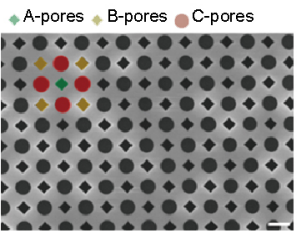

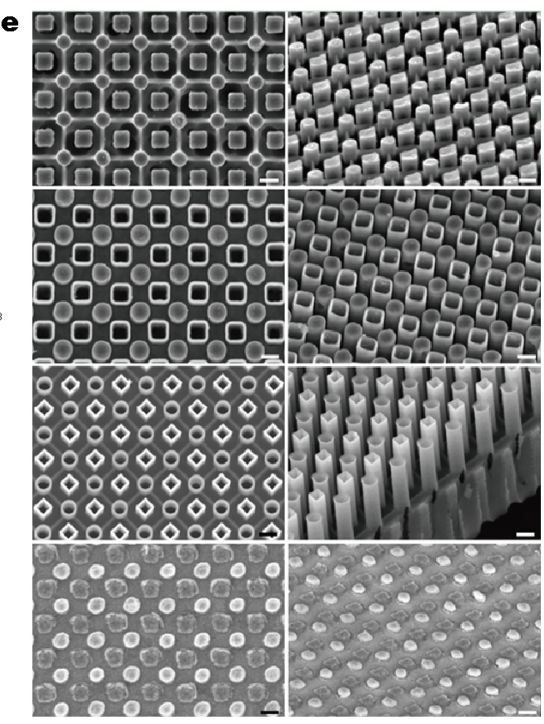

Figure 1 (a) Photo and SEM image of a typical binary-pore AAO template (A-pore, yellow square; B-pore, green circle) with a double-side barrier layer profile (indicated by yellow and green arrows, respectively). (b) Tuning B-pore shape with a combination of selective etching ( $30 \mathrm{~min}$ ) and pore widening: $2.5 \mathrm{~h}$ pore widening for four-cross B-pores (left) and $3.5 \mathrm{~h}$ pore-widening for eight-cross B-pores (right). (c) Left: three-dimensional reconstruction of $\mathrm{a} \mathrm{TiO}_{2}$ filled A-pore template with in-situ focused ion beam (FIB) milling (A-pores, purple; inverted pyramid voids, green). Right: COMSOL simulated electric field distributions on an imprinted $\mathrm{Al}$ foil with square arranged pattern at the very early stage of anodization. (d) SEM images of ternary-pore templates with different geometry combinations, where the anodized A- and B-pores are labelled with green and yellow squares and the selectively etched C-pores are labelled with red ovals (or circles), respectively. (e) Top-down and tilted SEM images of different binary materials (from top to bottom): $\mathrm{Ag}$ nanowire/Ni nanowire; $\mathrm{Cu}_{2} \mathrm{O}$ nanowire/TiO 2 nanotube; $\mathrm{TiO}_{2}$ nanotube $/ \mathrm{SnO}_{2}$ nanotube; $\mathrm{Au}$ nanodot/Ag nanodot, respectively. Scale bars, $200 \mathrm{~nm}$. Reprinted with permission from Ref. [8], Copyright 2017, Nature Publishing Group.

vices, and vertical nanowire transistors, showing superior properties in contrast to their corresponding single-component counterparts.

It is well known that AAO template has been widely used for fabrication of nanostructured materials [10,11]. However, as a significant breakthrough in the fabrication of binary materials, this reviving but unique binary-pore AAO template developed by Lei et al. [8] allows us to quantitatively disentangle the interplay between the sub-components and pursue oriented collective properties. It shall be expected that this nice piece of work will stimulate more efforts towards creating a new generation of multi-functional and innovative devices/systems based on differently desirable binary materials.

Received 5 April 2017; accepted 5 April 2017; published online 17 April 2017

1 Su B, Guo W, Jiang L. Learning from Nature: binary cooperative complementary nanomaterials. Small, 2015, 11: 1072-1096

2 Liu M, Jiang L. Dialectics of nature in materials science: binary cooperative complementary materials. Sci China Mater, 2016, 59:
239-246

3 Shevchenko EV, Talapin DV, Kotov NA, et al. Structural diversity in binary nanoparticle superlattices. Nature, 2006, 439: 55-59

4 Dong A, Chen J, Vora PM, et al. Binary nanocrystal superlattice membranes self-assembled at the liquid-air interface. Nature, 2010, 466: 474-477

5 Ye X, Zhu C, Ercius P, et al. Structural diversity in binary superlattices self-assembled from polymer-grafted nanocrystals. Nat Commun, 2015, 6: 10052

6 Shegai T, Chen S, Miljković VD, et al. A bimetallic nanoantenna for directional colour routing. Nat Commun, 2011, 2: 481

7 Liu N, Tang ML, Hentschel M, et al. Nanoantenna-enhanced gas sensing in a single tailored nanofocus. Nat Mater, 2011, 10: 631-636

8 Wen L, Xu R, Mi Y, et al. Multiple nanostructures based on anodized aluminium oxide templates. Nat Nanotech, 2017, 12: 244-250

9 Lei Y, Yang S, Wu M, et al. Surface patterning using templates: concept, properties and device applications. Chem Soc Rev, 2011, 40: $1247-1258$

10 Routkevitch D, Bigioni T, Moskovits M, et al. Electrochemical fabrication of CdS nanowire arrays in porous anodic aluminum oxide templates. J Phys Chem, 1996, 100: 14037-14047

11 Hulteen JC, Martin CR. A general template-based method for the preparation of nanomaterials. J Mater Chem, 1997, 7: 1075-1087 